\title{
Dynamic Contrast-Enhanced Magnetic Resonance Imaging Suggests Normal Perfusion in Normal-Appearing White Matter in Multiple Sclerosis
}

\author{
Michael Ingrisch, PhD, * Steven Sourbron, PhD, † Sina Herberich, MD, $\neq$ \\ Moritz Jörg Schneider, Dipl-Phys, MSc, * Tania Kümpfel, MD, § Reinhard Hohlfeld, MD, \&/I \\ Maximilian F. Reiser, MD, + and Birgit Ertl-Wagner, MD $\neq$
}

Objectives: Multiple sclerosis (MS) is a chronic, inflammatory disease of the central nervous system and has been associated with reduced perfusion in normal-appearing white matter (NAWM). The magnitude of this hypoperfusion is unclear. The present study aims to quantify NAWM perfusion with dynamic contrast-enhanced (DCE) magnetic resonance imaging (MRI) in patients with relapsing-remitting (RR) MS and in a control group.

Materials and Methods: The statistical power of a DCE-MRI acquisition to reveal hypoperfusion in MS was estimated using a Monte Carlo simulation: synthetic tissue curves with a contrast-to-noise ratio of 8 were generated for MS patients and control group using perfusion values reported in previous studies. A compartment-uptake model was fitted to these curves, yielding estimates of cerebral blood flow (CBF), cerebral blood volume (CBV), and permeability-surface area product (PS). This was repeated 1000 times. Mean and standard deviation of the resulting distributions were used to calculate the statistical power of a DCE-MRI study to detect perfusion differences between 16 control subjects and 24 MS subjects.

In an institutional review board-approved study, patients with RR-MS ( $\mathrm{n}=24$; mean age, 36 years; 17 women, mean Enhanced Disability Status Scale score, 3.25) and patients without history or symptoms of neurological disorder $(\mathrm{n}=16$; mean age, 49 years; 9 women) underwent a DCE-MRI examination with a previously established MRI protocol (3D SPGR sequence; 2.1 seconds temporal resolution; 44 slices; spatial resolution, $1.7 \times 1.7 \times 3 \mathrm{~mm}$ ). Regions were defined manually in the middle cerebral artery; in the frontal, periventricular, and occipital NAWM; in the pons; and in the thalamus, and CBF, CBV, and PS were quantified using a compartment-uptake model.

Parameter differences between MS and control groups were evaluated using a mixed linear model with subjects as random effect and controlling for age and sex. A $P$ value of less than 0.05 was considered to indicate statistical significance. Results: For all but one of previously reported effect sizes, the simulation study estimated a statistical power of $80 \%$ to $100 \%$ to detect reduced CBF in MS. In the patient study, mean (standard deviation) CBF in NAWM was 11.0 (15.1) and 10.4 (8.2) $\mathrm{mL} / 100 \mathrm{~mL}$ per minute in the MS and control groups, respectively. Mean CBV in NAWM was $0.50(0.45) \mathrm{mL} / 100 \mathrm{~mL}$ in the MS group and $0.48(0.28)$ $\mathrm{mL} / 100 \mathrm{~mL}$ in the control group. Mean values of PS in NAWM were $0.002 \mathrm{~mL}$

Received for publication June 13, 2016; and accepted for publication, after revision, July 8,2016.

From the * Josef Lissner Laboratory for Biomedical Imaging, Institute for Clinical Radiology, Ludwig-Maximilians-University Hospital Munich, Munich, Germany; $\dagger$ Leeds Institute of Cardiovascular and Metabolic Medicine, University of Leeds, Leeds, United Kingdom; $¥$ Institute for Clinical Radiology, and §Institute of Clinical Neuroimmunology, Ludwig-Maximilians-University Hospital; and $\|$ Munich Cluster for Systems Neurology (SyNergy), Munich, Germany.

Conflicts of interest and sources of funding: The work of R.H. was partially supported by DFG (SFB TR128), the Munich Cluster for Systems Neurology (ExC 1010 SyNergy), the Clinical Competence Network for Multiple Sclerosis, and the Verein zur Therapieforschung für Multiple-Sklerose-Kranke.

The authors report no conflicts of interest.

Correspondence to: Michael Ingrisch, PhD, Josef Lissner Laboratory for Biomedical Imaging, Institute for Clinical Radiology, Ludwig-Maximilians-University Hospital Munich, Marchioninistraße 15, 81377 Munich, Germany. E-mail: michael.ingrisch@med.lmu.de.

Copyright (C) 2016 Wolters Kluwer Health, Inc. All rights reserved.

ISSN: 0020-9996/17/5203-0135

DOI: $10.1097 /$ RLI.0000000000000320
$(0.027) / 100 \mathrm{~mL}$ per minute in the control group and $-0.001(0.015) \mathrm{mL} / 100 \mathrm{~mL}$ per minute in the MS patients. Differences between patient groups were not statistically significant for $\mathrm{CBF}, \mathrm{CBV}$, mean transit time, and $\mathrm{PS}(P=0.44, P=0.20$, $P=0.78, P=0.66$, respectively). In both groups, the influence of age on any parameter was nonsignificant. Cerebral blood flow and CBV in the thalamus and pons were significantly higher than in NAWM regions $(P<1 \mathrm{e}-4)$; mean transit time was significantly shorter than in NAWM $(P<1 \mathrm{e}-4)$. Permeability-surface area product was not significantly different from zero $(P>0.25)$ in all evaluated regions. Conclusions: Despite high statistical power, we could not confirm previous reports of NAWM hypoperfusion in MS. This indicates that, at least in our patient cohort, potential hypoperfusion is much less pronounced than reported in previous studies.

Key Words: dynamic contrast-enhanced MRI, multiple sclerosis, normal-appearing white matter

(Invest Radiol 2017;52: 135-141)

$\mathrm{M}$ ultiple sclerosis (MS) is a chronic, inflammatory autoimmune disease of the central nervous system. The disease often follows a relapsing-remitting (RR) course, with focal inflammatory demyelinating lesions being one of the hallmarks of the disorder. In addition, MS has also been reported to be associated with a diffuse reduction of perfusion, that is, the blood supply of tissue, in normal-appearing white matter (NAWM). Early studies using single photon emission computed tomography or positron emission tomography ${ }^{1-3}$ reported a significant reduction of cerebral blood flow (CBF) in the NAWM of patients with MS as compared with healthy control groups. Others have used a contrast agent-based magnetic resonance imaging (MRI) technique (dynamic susceptibility-contrast [DSC] MRI) and demonstrated a pronounced reduction of cerebral perfusion in patients with $\mathrm{MS}$, as compared with healthy controls, ${ }^{4-7}$ with differences of up to $100 \%$. These findings have led to speculations as to whether this hypoperfusion is a cause or a consequence of $\mathrm{MS},{ }^{8}$ and potential therapy pathways have also been suggested, ${ }^{9}$ highlighting the importance of cerebral hypoperfusion in MS as a potential therapeutic target. ${ }^{10}$ The magnitude of this hypoperfusion, however, is unclear. A perfusion difference of only $20 \%$ was reported in a study using arterial spin labeling ${ }^{9}$ (ASL), whereas another study ${ }^{11}$ even identified a subgroup of patients with "highly inflammatory" MS where NAWM perfusion was not reduced but increased.

In the present work, we aimed to investigate NAWM perfusion with dynamic contrast-enhanced (DCE) MRI, based on a timeresolved T1-weighted measurement of the signal enhancement during and after the administration of contrast agent. Dynamic contrastenhanced-MRI is a well-established technique for the measurement and quantification of extracerebral tissue perfusion and has also been used for the measurement of hemodynamics in inflammatory lesions and in NAWM in patients with MS. ${ }^{12}$ Initially, we performed a simulation study to estimate the statistical power of DCE-MRI to detect cerebral hypoperfusion. Subsequently, we conducted a patient study in which we compared NAWM perfusion in a group of patients with RR-MS to a group of patients without an inflammatory or demyelinating disorder of the brain. 


\section{MATERIALS AND METHODS}

\section{Simulations and Power Analysis}

To assess the effect size and the statistical power of a DCE-MRI study, a Monte Carlo simulation was performed as follows: values for $\mathrm{CBF}$ and cerebral blood volume (CBV) were drawn from normal distributions with mean values and standard deviations from previous

TABLE 1. Patient Demographics

\begin{tabular}{|c|c|c|c|c|c|c|c|}
\hline $\begin{array}{l}\text { Subject } \\
\text { ID }\end{array}$ & $\begin{array}{l}\text { Patient } \\
\text { Group }\end{array}$ & Sex & $\begin{array}{c}\text { Age, } \\
\mathbf{y}\end{array}$ & $\begin{array}{c}\text { Duration } \\
\text { of } \\
\text { Disease, } y\end{array}$ & $\begin{array}{c}\text { Time } \\
\text { Since } \\
\text { Last } \\
\text { Relapse, } \\
\text { mo }\end{array}$ & EDSS & $\begin{array}{c}\text { No. } \\
\text { Contrast- } \\
\text { Enhancing } \\
\text { Lesions }\end{array}$ \\
\hline 1 & MS & $\mathrm{M}$ & 23 & 2 & 1 & 4.5 & 0 \\
\hline 4 & MS & $\mathrm{F}$ & 51 & 11 & 6 & 4 & 0 \\
\hline 5 & MS & $\mathrm{F}$ & 31 & 5 & 126 & 5 & 0 \\
\hline 6 & MS & $\mathrm{F}$ & 43 & 13 & 0 & 6 & 3 \\
\hline 7 & MS & $\mathrm{F}$ & 38 & 5 & 36 & 2 & 0 \\
\hline 8 & MS & $\mathrm{M}$ & 31 & 12 & 96 & 2 & 0 \\
\hline 10 & MS & $\mathrm{M}$ & 31 & 0.3 & 1 & 1 & 0 \\
\hline 11 & MS & $\mathrm{F}$ & 30 & 0.3 & 2 & 1.5 & 5 \\
\hline 13 & MS & $\mathrm{M}$ & 30 & 14 & 5 & 3 & 3 \\
\hline 16 & MS & $\mathrm{M}$ & 47 & 7 & 1 & 4 & 0 \\
\hline 17 & MS & $\mathrm{M}$ & 56 & 11 & 7 & 6 & 0 \\
\hline 19 & MS & $\mathrm{F}$ & 34 & 12 & 137 & 3 & 0 \\
\hline 20 & MS & $\mathrm{F}$ & 46 & 21 & 0 & 6.5 & 0 \\
\hline 22 & MS & $\mathrm{F}$ & 23 & 3 & 11 & 0 & 1 \\
\hline 25 & MS & $\mathrm{F}$ & 48 & 4 & 5 & 2.5 & 0 \\
\hline 26 & MS & $\mathrm{F}$ & 28 & 10 & 1 & 4 & 1 \\
\hline 29 & MS & $\mathrm{F}$ & 55 & 11 & 9 & 4 & 1 \\
\hline 30 & MS & $\mathrm{F}$ & 30 & 0.5 & 5 & 2 & 0 \\
\hline 31 & MS & $\mathrm{F}$ & 42 & 12 & 0 & 4 & 0 \\
\hline 32 & MS & $\mathrm{M}$ & 21 & 0.8 & 32 & 0 & 2 \\
\hline 33 & MS & $\mathrm{F}$ & 28 & 2 & 8 & 2 & 0 \\
\hline 37 & MS & $\mathrm{F}$ & 33 & 0.5 & 1 & 4 & 0 \\
\hline 40 & MS & $\mathrm{F}$ & 40 & 4 & 8 & 3 & 2 \\
\hline 44 & MS & $\mathrm{F}$ & 24 & 6 & 1 & 4 & 0 \\
\hline 2 & Control & $\mathrm{F}$ & 20 & & & & \\
\hline 3 & Control & $\mathrm{F}$ & 52 & & & & \\
\hline 9 & Control & $\mathrm{M}$ & 52 & & & & \\
\hline 15 & Control & $\mathrm{M}$ & 67 & & & & \\
\hline 18 & Control & $\mathrm{F}$ & 30 & & & & \\
\hline 21 & Control & $\mathrm{F}$ & 65 & & & & \\
\hline 23 & Control & $\mathrm{M}$ & 38 & & & & \\
\hline 24 & Control & $\mathrm{F}$ & 58 & & & & \\
\hline 27 & Control & $\mathrm{M}$ & 48 & & & & \\
\hline 28 & Control & $\mathrm{F}$ & 32 & & & & \\
\hline 34 & Control & $\mathrm{M}$ & 69 & & & & \\
\hline 36 & Control & $\mathrm{F}$ & 57 & & & & \\
\hline 39 & Control & $\mathrm{F}$ & 63 & & & & \\
\hline 45 & Control & $\mathrm{M}$ & 26 & & & & \\
\hline 46 & Control & $\mathrm{F}$ & 45 & & & & \\
\hline 48 & Control & $\mathrm{M}$ & 66 & & & & \\
\hline
\end{tabular}

Age and duration of disease are provided in years, time since last relapse in months. EDSS indicates Enhanced Disability Status Scale; MS, multiple sclerosis; $\mathrm{M}$, male; F, female.

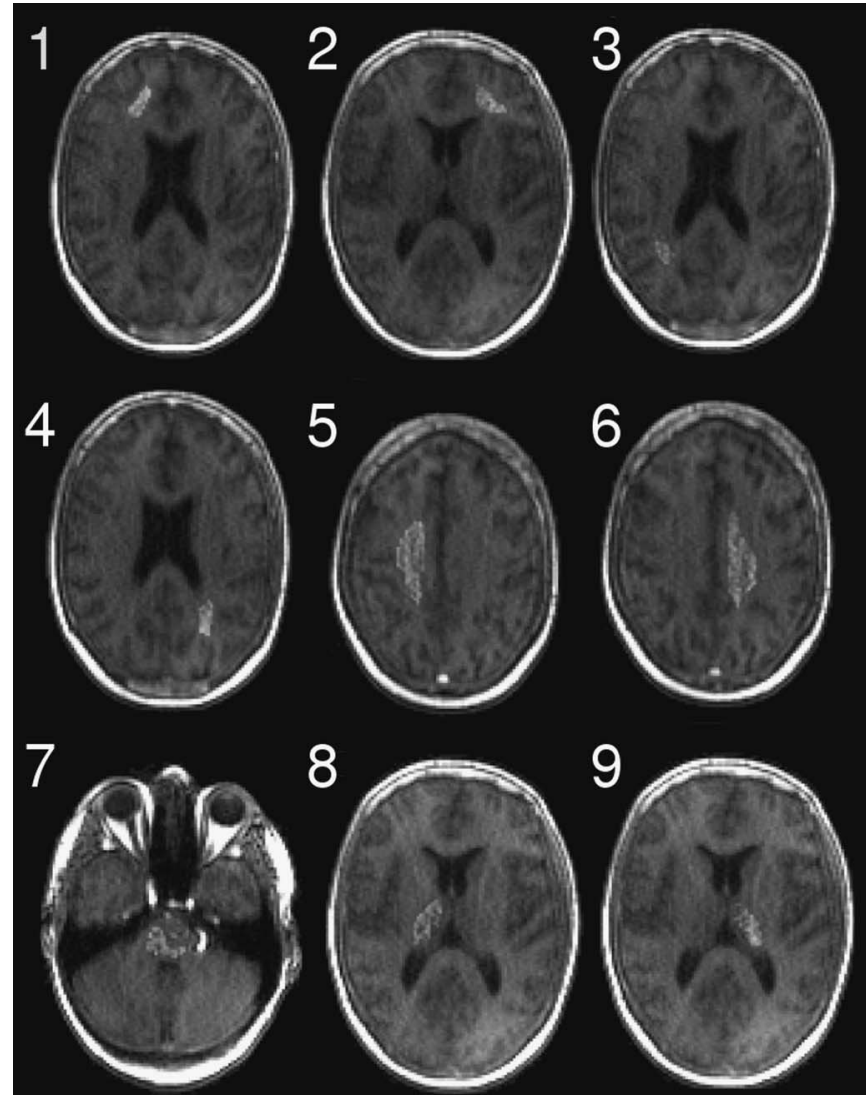

FIGURE 1. Representative example of defined tissue regions in the frontal (1 and 2), occipital (3 and 4), and periventricular (5 and 6) NAWM; in the pons (7); and in the thalamus ( 8 and 9 ). Whenever possible, regions were defined on 3 adjacent slices to increase region size. Figure 1 can be viewed online in color at www.investigativeradiology.com.

studies $^{1,4,5,9}$; Permeability-surface area product (PS) was assumed to be zero. Using these draws, synthetic tissue signal curves for NAWM in MS and control patients were generated using an arterial input function (AIF) from a pilot study ${ }^{12}$ with a compartment-uptake model. To these curves, Gaussian noise was added to achieve a contrast-to-noise ratio $(\mathrm{CNR})$ similar to the pilot study $^{12}(\mathrm{CNR}=8)$. To the resulting curve, a compartment-uptake model ${ }^{13}$ was fitted, yielding estimates of CBF, CBV, mean transit time (MTT), and PS. This process was repeated for 1000 draws to estimate the parameter distributions. Mean and standard deviation of the resulting distributions were used to calculate effect sizes, estimated as Cohen $\mathrm{d},{ }^{14}$ and the statistical power of a DCE-MRI study to detect perfusion differences with 16 subjects in the control group and 24 subjects in the MS group.

\section{Patients and Imaging}

The institutional review board approved this study, and informed consent was obtained from all participants; methods were carried out in accordance with the approved guidelines. Twenty-four consecutive patients with confirmed RR-MS ( $\mathrm{n}=24$; mean age, 36 years; range, 21-56; 17 women; mean Enhanced Disability Status Scale score, 3.25) with a clinical indication for a contrast-enhanced MRI examination were included in the study. Exclusion criteria were relapse at the time point of imaging or glucocorticoid therapy in the 4 weeks before imaging. As a control group, patients without history or symptoms of neurological disorder ( $\mathrm{n}=16$; mean age, 49 years; range, 20-69; 9 women) were included; the patients in this control cohort presented for the imaging evaluation of a hearing disorder to rule out vestibular schwannoma. 

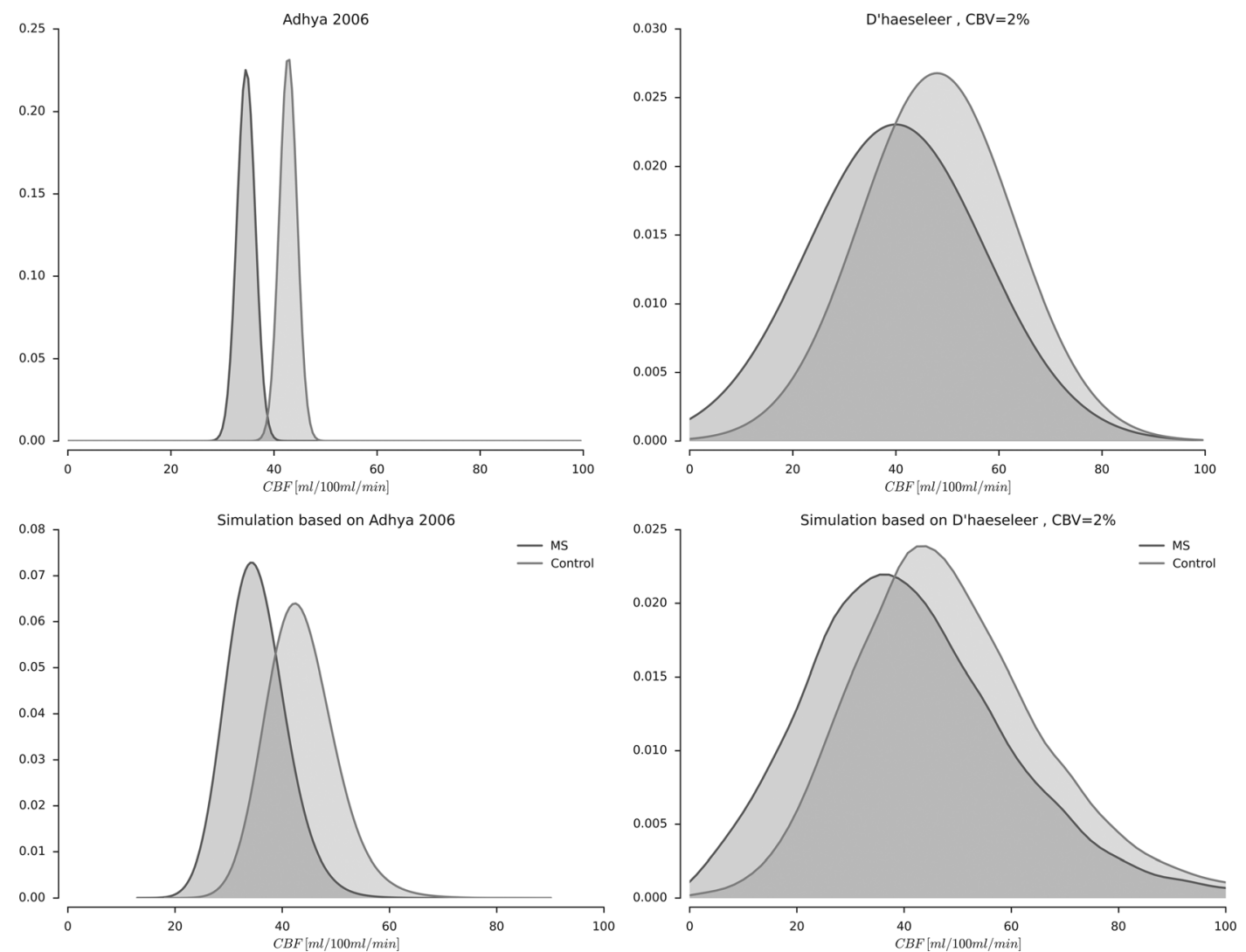

FIGURE 2. Normal distributions of CBF in MS and in control patients, as reported in Adhya et $\mathrm{al}^{5}$ (top left) and in $\mathrm{D}$ 'haeseleer et al ${ }^{9}$ (top right). The corresponding effect sizes are $d=4.92$ (top left) and $d=0.51$ (top right). After a simulated DCE-MRI measurement, distributions appear broader and show more overlap (bottom row), resulting in reduced effect sizes of $d=1.3$ (bottom left) and $d=0.09$ (bottom right), respectively. Figure 2 can be viewed online in color at www.investigativeradiology.com.

Exclusion criteria for both cohorts were a history of other neurological disorders other than MS in the MS cohort and hearing loss in the control cohort, the presence of an intracranial tumor, as well as usual contraindications for MRI, including cardiac pacemakers, other ferromagnetic implants, claustrophobia, and pregnancy. Detailed patient characteristics are shown in Table 1.

All measurements were performed on a clinical $3 \mathrm{~T}$ scanner (Magnetom Verio; Siemens Healthcare, Erlangen, Germany). The imaging protocol included precontrast 3D T1-weighted sequences, 3D
FLAIR sequences, and axial T2-weighted sequences as well as contrast-enhanced 3D T1-weighted sequences. One hundred seventy DCE-MRI volumes were acquired during a total acquisition time of 6 minutes with a T1-weighted 3D spoiled gradient echo sequence with a coverage of the full brain at a spatial resolution of $1.7 \times 1.7 \times 3 \mathrm{~mm}^{3}$ and a temporal resolution of 2.1 seconds per volume, as described previously ${ }^{12}$ (TE/TR, 0.85/2.29 milliseconds; flip angle, 19 degrees; bandwidth, $1220 \mathrm{~Hz} /$ pixel; field of view, $220 \times 178 \times 132 \mathrm{~mm}$; matrix size, $128 \times 104 \times 44$; parallel imaging with acceleration factor 2 ; TWIST

TABLE 2. Overview of Sample Sizes, Reported CBF Values (Mean and Standard Deviation in Both Groups), Reported Effect Sizes, Simulated Effect Sizes, and Estimated Statistical Power After a DCE Measurement With 16 Control Patients and 24 MS Patients

\begin{tabular}{|c|c|c|c|c|c|c|c|c|}
\hline & Method & $\mathbf{n}_{\mathbf{M S}}$ & $\mathbf{n}_{\text {control }}$ & $\begin{array}{c}\text { CBF } \mathrm{MS}, \mathrm{mL} / 100 \mathrm{~mL} \\
\text { per minute }\end{array}$ & $\begin{array}{c}\text { CBF Control, } \mathrm{mL} / 100 \mathrm{~mL} \\
\text { per minute }\end{array}$ & $\begin{array}{l}\text { Original Effect } \\
\text { Size }\end{array}$ & $\begin{array}{c}\text { Simulated Effect } \\
\text { Size }\end{array}$ & $\begin{array}{c}\text { Estimated } \\
\text { Power }\end{array}$ \\
\hline Brooks et al, ${ }^{1} 1985$ & PET & 15 & 13 & $21(4)$ & $27(5)$ & 1.39 & 1.23 & 0.92 \\
\hline Sun et al, ${ }^{2} 1998$ & PET & 20 & 14 & $17.7(5.0)$ & $23.9(5.6)$ & 1.22 & 1.03 & 0.81 \\
\hline Law et $\mathrm{al}^{4}{ }^{4} 2004$ & DSC & 17 & 17 & $16.3(9.5)$ & $34.8(8.4)$ & 2.14 & 2.02 & 1.00 \\
\hline Adhya et al, ${ }^{5} 2006$ & DSC & 11 & 11 & $34.6(1.8)$ & $42.8(1.7)$ & 4.92 & 1.65 & 0.99 \\
\hline $\begin{array}{l}\text { D'haeseleer et } \text { al }^{9}{ }^{9} \\
2013\end{array}$ & ASL & 15 & 15 & $40.0(17.3)$ & $48.0(14.9)$ & 0.51 & 0.03 & 0.05 \\
\hline
\end{tabular}

In the study by D'haeseleer et al, no CBV values were reported, so that the simulation was performed with an assumed CBV value $2 \%$.

CBF indicates cerebral blood flow; DCE, dynamic contrast-enhanced; MS, multiple sclerosis; PET, positron emission tomography; DSC, dynamic susceptibility-contrast; ASL, arterial spin labeling. 
view sharing ${ }^{15}$ with $\left.\mathrm{pA} / \mathrm{pB}=0.25 / 0.22\right)$. Ten seconds after the DCE acquisition was started, a standard dose of contrast agent $(0.1 \mathrm{mmol} / \mathrm{kg}$ body weight of gadobutrol, Bayer Schering) was injected intravenously at a rate of $3 \mathrm{~mL} / \mathrm{s}$. The injection was split into 2 injections separated by 60 seconds to reduce the peak concentration during the first pass of the contrast agent; each injection was followed by a flush of $30 \mathrm{~mL}$ saline solution with the same injection rate.

\section{Image Analysis and Quantification}

Structural MRI sequences of both cohorts were reviewed by a neuroradiologist (B.E.W.) with more than 15 years of experience in neuroimaging to rule out vestibular or cochlear schwannomas, other intracranial tumors, cerebral hemorrhage, cerebral microbleeds, hydrocephalus, acute or subacute cerebral ischemia, and other diseases that may affect cerebral perfusion.

Image postprocessing was done using in-house written software $^{16}$ as described previously. ${ }^{12}$ To aid the definition of evaluation regions, maps of the average baseline signal $S_{0}$ before the arrival of contrast agent and of the area under the curve (AUC) of the signal enhancement $S(t)-S_{0}$ were calculated.

The AIF was measured in the middle cerebral artery. A rectangular region encompassing the middle cerebral artery was defined manually on the AUC maps; the 6 voxels with the highest AUC values within this region were used to measure the signal enhancement course in the artery. To correct for partial volume effects, a venous output curve was measured in the single pixel with the highest AUC value in the sagittal sinus (SS).

For the assessment of perfusion, tissue regions were defined bilaterally in the frontal, periventricular, and occipital white matter; in the thalami; and in the pons (see Fig. 1). For the definition of these regions, the map of the baseline signal was used. In the MS cohort, FLAIR images were visually coregistered to the baseline map to ensure that no FLAIR-hyperintense areas were included in the NAWM regions. Whenever possible, regions were defined on consecutive slices to increase region size.

Averaged time-intensity curves were calculated for all previously defined regions and converted to contrast agent concentration using the absolute signal enhancement $S(t)-S_{0}$. The absolute signal enhancement is proportional to the contrast agent concentration and, unlike the relative signal enhancement, does not depend on the precontrast relaxation time. ${ }^{13}$ The AIF was corrected for partial volume effects using the reference measurement in the $\mathrm{SS} .{ }^{12}$ Briefly, the apparent blood volume in the SS was determined by deconvolving the venous curve with the measured AIF, which is affected by partial volume effects. These partial volume effects cause an overestimation of the apparent venous blood volume. Because the true blood volume in the sinus sagittalis voxel can be assumed to be $100 \%$, a correction factor for the AIF can be calculated. ${ }^{17}$ This correction factor was then used to rescale the AIF. The arterial plasma concentration was derived by scaling the arterial blood concentration with $1 / 1(1-h c t)$, where hct is the hematocrit value. A constant hematocrit of $h c t=0.45$ was assumed because no individual hematocrit values were available.

A compartment uptake model ${ }^{13}$ was then fitted to the signal enhancement curves in each region, yielding estimates of $\mathrm{CBF}, \mathrm{CBV}$, MTT, and PS.

\section{Statistical Analysis}

Statistical evaluation of the data was performed in $\mathrm{R}^{18}$ using the packages lme 4 and lmerTest. ${ }^{19,20}$ Differences in CBF, CBV, and PS between MS patients and the control group were evaluated using a linear mixed model with subjects as random effect. Each perfusion parameter was modeled separately. The influence of duration of disease, number of contrast-enhancing lesions, and time since last relapse were included into the model and tested for statistical relevance. Fixed effects of the final model were group, age, sex, and region (frontal, occipital, and periventricular NAWM; thalamus; pons). A $P$ value of less than 0.05 was considered to indicate statistical significance.

In an additional analysis, patients with contrast-enhancing lesions were excluded and the evaluation was repeated.

\section{RESULTS}

\section{Simulations and Power Analysis}

The simulated DCE-MRI acquisition resulted in a broadening compared with the original parameter distributions based on the reported parameter values, due to the low CNR that is typical for DCE-MRI in the presence of an intact blood-brain barrier (BBB). ${ }^{12,17}$ Original and simulated $\mathrm{CBF}$ distributions for 2 selected studies are shown in Figure 2. Based on the resulting effect sizes, a DCE-MRI study with 16 patients in the control group and 24 patients in the MS group has a statistical power of $99 \%$ to discriminate $\mathrm{CBF}$ between the 2 patient groups for $\mathrm{CBF}$ differences as reported in a DSC study. ${ }^{5}$ For the CBF differences from a recent ASL study, ${ }^{9}$ displayed top right in Figure 2, the statistical power reduces to $6 \%$ for the same sample sizes. Reported and simulated effect sizes from several earlier studies, along with the estimated statistical power of a DCE-MRI study, are displayed in Table 2. For most

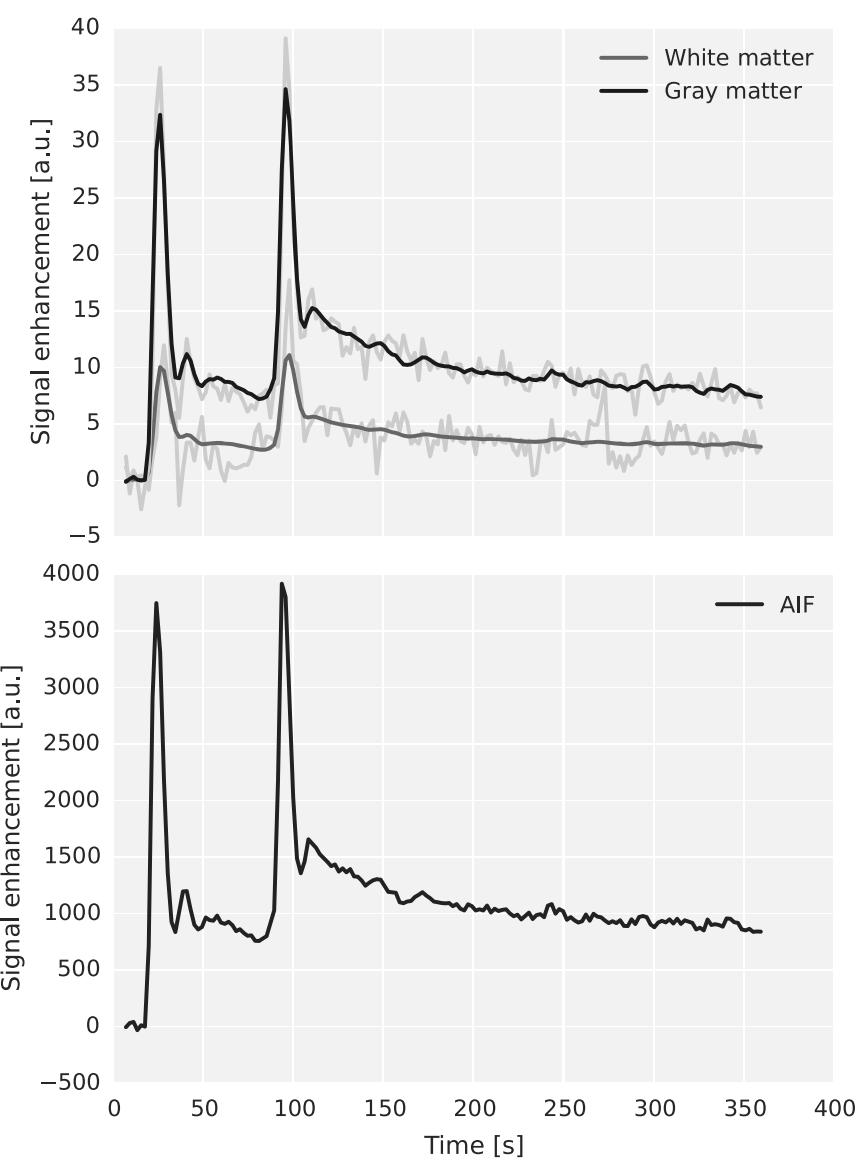

FIGURE 3. Top: Exemplary signal enhancement curves from a patient with MS (subject 44) in gray and white matter curves, along with model fits. The white matter curve was measured in a region in the occipital white matter, with $\mathrm{CBF}=12.7 \mathrm{~mL} / 100 \mathrm{~mL}$ per minute, $\mathrm{CBV}=0.64 \mathrm{~mL} /$ $100 \mathrm{~mL}$, and $\mathrm{PS}=0.001 \mathrm{~mL} / 100 \mathrm{~mL}$ per minute. The gray matter curve was measured in the left thalamus, the model fit yielded $\mathrm{CBF}=74.2 \mathrm{~mL} /$ $100 \mathrm{~mL}$ per minute, $\mathrm{CBV}=2.7 \mathrm{~mL} / 100 \mathrm{~mL}$, $\mathrm{MTT}=1.4 \mathrm{~s}$, and $\mathrm{PS}=-0.02 \mathrm{~mL} / 100 \mathrm{~mL}$ per minute. Bottom: the corresponding AIF. Both plots display the absolute signal enhancement. Figure 3 can be viewed online in color at www.investigativeradiology.com. 

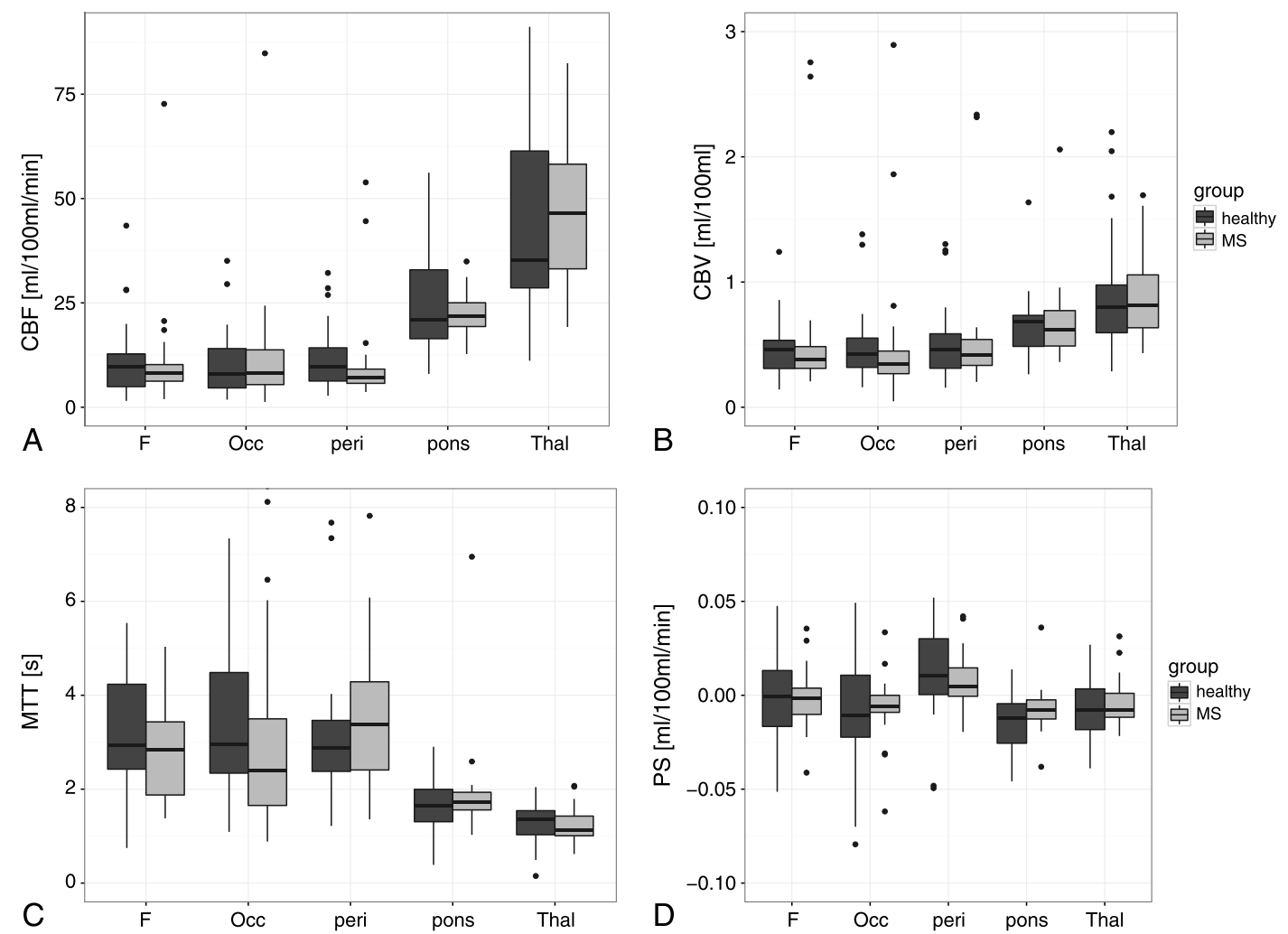

FIGURE 4. Overview of determined values of CBF (A) given in $\mathrm{mL} / 100 \mathrm{~mL}$ per minute, CBV (B) given in $\mathrm{mL} / 100 \mathrm{~mL}, \mathrm{MTT}(\mathrm{C})$ in seconds, and PS (D) in $\mathrm{mL} / 100 \mathrm{~mL}$ per minute in patients and control group in all evaluated regions. The centerline of the boxes indicates the median; the boxes indicate the range from the 25 th to 75 th percentile; the whiskers extend to the highest value within $1.5^{\star}$ interquartile range; values outside the whiskers are plotted as dots.

previously reported effect sizes, the estimated statistical power of a DCEMRI study is larger than $80 \%$.

\section{Quantification}

Representative tissue curves for gray matter and NAWM, measured in a patient with MS (subject 44), are shown in Figure 3. The characteristic dual-peak form of both curves results from the double injection of contrast agent. The amplitude of the NAWM curve is much smaller, reflecting the lower values of $\mathrm{CBF}$ and $\mathrm{CBV}$.

Quantitative parameter estimates of CBF, CBV, and PS are summarized in Figure 4. Cerebral blood flow and CBV were increased in the pons and thalamus, as compared with NAWM, and are similar in MS patients and control group (see Fig. 4). Likewise, the PS does not demonstrate obvious differences between groups. The mean CBF in NAWM was 11.0 (15.1) and $10.4(8.2) \mathrm{mL} / 100 \mathrm{~mL}$ per minute in the MS and control groups, respectively. The mean CBV in NAWM was $0.50(0.45) \mathrm{mL} / 100 \mathrm{~mL}$ in the MS group and $0.48(0.28) \mathrm{mL} / 100 \mathrm{~mL}$ in the control group. The mean values of PS in NAWM were $0.002 \mathrm{~mL}(0.027) \mathrm{mL} / 100 \mathrm{~mL}$ per minute in the control group and $-0.001(0.015) \mathrm{mL} / 100 \mathrm{~mL}$ per minute in the MS patients.

Differences between patient groups were not statistically significant for CBF, CBV, MTT, and PS $(P=0.44, P=0.20, P=0.78$, $P=0.66$, respectively). Sex had no significant influence on either of these parameters; in both groups, the influence of age on any parameter was nonsignificant. Cerebral blood flow and CBV in the thalamus and pons were significantly higher than in NAWM regions $(P<1 \mathrm{e}-4)$; MTT was significantly shorter than in NAWM $(P<1 \mathrm{e}-4)$. Permeabilitysurface area product was not significantly different from zero $(P>0.25)$ in all evaluated regions. Medication, total duration of disease, number of contrast-enhancing lesions, and time since last relapse had no significant influence and were therefore not included in the final statistical model. Excluding the 8 patients with active, contrast-enhancing lesions from the statistical analysis did not alter the qualitative outcome of the statistical analysis; in particular, no significant differences between patient groups could be observed.

\section{DISCUSSION}

In this study, perfusion parameters in NAWM in patients with RR-MS were compared with a control group to assess whether previous findings of reduced NAWM perfusion in RR-MS could be confirmed by means of DCE-MRI. The simulation study demonstrated that our DCE-MRI study has sufficient statistical power to discriminate previously reported large CBF differences, whereas small CBF differences, as reported in a recent study, may be missed due to low statistical power. In our patient study, we were not able to detect statistically significant differences between patients with RR-MS and a control group regarding $\mathrm{CBF}, \mathrm{CBV}$, and PS in NAWM and in gray matter.

These results are not in line with previous reports that studied NAWM perfusion in MS. Early nuclear medicine studies ${ }^{1-3}$ have found a significantly reduced CBF in patients with MS. These observations were confirmed by several publications, ${ }^{4-7}$ who, albeit with partially overlapping ${ }^{7}$ patient collectives, reported CBF to be markedly reduced in RR-MS. A recent study ${ }^{9}$ employed yet another technique, ASL, and found a less pronounced but still significant $\mathrm{CBF}$ reduction in patients with RR-MS. We performed Monte Carlo simulations with values reported in these studies and demonstrated a DCE-MRI study to have high $(>80 \%)$ statistical power to discriminate NAWM hypoperfusion for all effect sizes but those reported in the ASL study (see Table 2). 
Taken together, these findings indicate that, in our patient group, NAWM hypoperfusion may either be nonexistent or must be notably less pronounced than reported in previous studies.

A recent study ${ }^{11}$ reported NAWM perfusion not only to be not reduced in a subgroup with "highly active" MS but even to be higher than in control patients. In this study, patients were followed up by monthly MRI examinations, and highly active was defined as a marked increase in the number of contrast-enhancing lesions. ${ }^{11}$ In our patient cohort, no follow-up data were available due to our cross-sectional study design, so that this definition of highly active MS could not be used. A surrogate marker of disease activity, the total number of contrast-enhancing lesions, was not associated with a difference in NAWM perfusion. In addition, no statistically significant differences in $\mathrm{CBF}$ or $\mathrm{CBV}$ between patient groups emerged when patients with contrast-enhancing lesions were excluded from the analysis. Nevertheless, the existence of MS subgroups with increased and reduced NAWM perfusion suggests that the role of NAWM perfusion in MS is not yet fully understood and requires further investigation.

A potential explanation for the discrepancy between early studies reporting a pronounced hypoperfusion and recent studies ${ }^{9,11}$ may be found in the "winner's course." 21 This is a statistical phenomenon by which the first studies to report a significant effect usually overestimate the effect size, resulting from a combination of small sample sizes and low statistical power. In the present study, we have derived estimates of the statistical power of our study design by means of a simulation study and demonstrated our study to have sufficient statistical power to detect previously reported large differences in NAWM perfusion.

We observed values for CBF and CBV that are well below those published in the literature. This underestimation is present in both patient groups, indicating a systematic effect. By using the absolute signal enhancement as approximation of the concentration, we have utilized a very simple, linear signal model. When large values for the concentration are reached, for example, during the first pass of contrast agent in the vessels, the underlying linearity assumption may be violated. This would, however, cause an underestimation of the AIF and hence an overestimation of $\mathrm{CBF}$ and $\mathrm{CBV}$, contrary to the observations. This leaves water exchange effects as a possible explanation for our observation. These effects are particularly relevant when large differences in relaxation rates occur, for example, during the first pass of contrast agent, where the intravascular contrast agent concentration and hence the relaxation rate is large. When not accounted for in the signal analysis, this leads to an underestimation of the contrast agent concentration, which, in turn, causes an underestimation of CBF and $\mathrm{CBV}^{22,23}$ The exact amount of this underestimation depends on several parameters of the pulse sequence that is used for acquisition, ${ }^{24,25}$ but also on the transendothelial water exchange rate. Utilizing a more complex signal model $^{23}$ would have accounted for both nonlinearities and water exchange effects, but would have required maps of precontrast relaxation rate and possibly flip angle; these data were not available in this study. In our study, we found PS to be zero in both patient groups; hence, we assume that the transendothelial water exchange is unlikely to be different between patient groups. Therefore, we do not expect that effects of slow water exchange influence the comparison of patient groups.

A major difficulty in perfusion MRI is the lack of standardization of data acquisition and quantification, partly due to the absence of an accepted criterion standard. Cerebral perfusion MRI has long been dominated by DSC MRI due to the large CNR, but absolute quantification of DSC remains challenging. ${ }^{26}$ In particular, in light of the recent discussion about gadolinium deposition in the brain, ${ }^{27-29}$ ASL is a very attractive technique for the assessment of cerebral perfusion because it does not require the administration of contrast agent. Although NAWM hypoperfusion in MS has been demonstrated recently by means of ASL, ${ }^{9}$ the detection of white matter perfusion abnormalities by ASL is seen critically. ${ }^{30}$ In the present work, we have chosen a DCE-MRI approach, which allowed us to measure CBF, CBV, and PS simultaneously, but this approach is limited by the low CNR that results from the low CBV in healthy brain tissue. An increase in CNR, for example, by using a larger voxel size, would be very helpful to detect subtle differences in perfusion below the detection limit of our study, but an upper limit on the voxel size is imposed by the need of measuring an AIF. Our modeling approach followed an emerging standardization of notation and tracer-kinetic modeling, ${ }^{31,32}$ thereby improving interstudy comparability.

Recently, subtle BBB leakage in NAWM has been reported in patients with MS. ${ }^{33}$ While our approach in principle allows for a measurement of PS as a surrogate marker of BBB leakage, our study was not optimized for this purpose. Because the focus of our study was to detect differences in CBF, we have not estimated the statistical power for the detection of increased PS. For a reliable measurement of subtle BBB leakage, a longer total acquisition time from $15^{34}$ to $25^{35} \mathrm{mi}$ nutes, combined with corrections for scanner drift and possibly image registration to account for patient movement, would have been benefi$\mathrm{cial}^{35}$; the total acquisition time in our study may not have been sufficient to resolve small values of PS.

Our study is not without limitations. The age distributions of the 2 patient groups are not fully matched. However, the statistical analysis of our data did not reveal a significant influence of age on cerebral perfusion in either group of our patient cohort. Moreover, the statistical model would have adjusted for potential effects of age. In addition, we were not able to classify our patients into "high-inflammatory" and "low-inflammatory"11 due to the crosssectional design of our study, which limited the respective subgroup analyses. Nevertheless, the exclusion of patients with CE-lesions did not alter the statistical results.

In conclusion, we were not able to confirm previous observations of NAWM hypoperfusion in MS in our patient groups, despite high statistical power for all but the most subtle previously reported differences. A possible explanation for this negative result is that - at least in our patient cohort-potential NAWM hypoperfusion is much less pronounced than reported in most previous studies.

\section{REFERENCES}

1. Brooks DJ, Leenders KL, Head G, et al. Studies on regional cerebral oxygen utilisation and cognitive function in multiple sclerosis. J Neurol Neurosurg Psychiatry. 1984;47:1182-1191.

2. Sun X, Tanaka M, Kondo S, et al. Clinical significance of reduced cerebral metabolism in multiple sclerosis: a combined PET and MRI study. Ann Nucl Med. 1998; 12:89-94.

3. Lycke J, Wikkelsö C, Bergh AC, et al. Regional cerebral blood flow in multiple sclerosis measured by single photon emission tomography with technetium-99m hexamethylpropyleneamine oxime. Eur Neurol. 1993;33:163-167.

4. Law M, Saindane AM, Ge Y, et al. Microvascular abnormality in relapsingremitting multiple sclerosis: perfusion MR imaging findings in normalappearing white matter. Radiology. 2004;231:645-652.

5. Adhya S, Johnson G, Herbert J, et al. Pattern of hemodynamic impairment in multiple sclerosis: dynamic susceptibility contrast perfusion MR imaging at $3.0 \mathrm{~T}$ Neuroimage. 2006;33:1029-1035.

6. Ge Y, Law M, Johnson G, et al. Dynamic susceptibility contrast perfusion MR imaging of multiple sclerosis lesions: characterizing hemodynamic impairment and inflammatory activity. AJNR Am J Neuroradiol. 2005;26: 1539-1547.

7. Inglese M, Adhya S, Johnson G, et al. Perfusion magnetic resonance imaging correlates of neuropsychological impairment in multiple sclerosis. J Cereb Blood Flow Metab. 2008;28:164-171.

8. Saindane AM, Law M, Ge Y, et al. Correlation of diffusion tensor and dynamic perfusion MR imaging metrics in normal-appearing corpus callosum: support for primary hypoperfusion in multiple sclerosis. AJNR Am J Neuroradiol. 2007; 28:767-772.

9. D'haeseleer M, Beelen R, Fierens Y, et al. Cerebral hypoperfusion in multiple sclerosis is reversible and mediated by endothelin-1. Proc Natl Acad Sci US A. 2013; 110:5654-5658. 
10. D'haeseleer M, Hostenbach S, Peeters I, et al. Cerebral hypoperfusion: a new pathophysiologic concept in multiple sclerosis? J Cereb Blood Flow Metab. 2015;35: 1406-1410.

11. Bester M, Forkert ND, Stellmann JP, et al. Increased perfusion in normal appearing white matter in high inflammatory multiple sclerosis patients. PLoS One. 2015;10:e119356.

12. Ingrisch $\mathrm{M}$, Sourbron $\mathrm{S}$, Morhard $\mathrm{D}$, et al. Quantification of perfusion and permeability in multiple sclerosis: dynamic contrast-enhanced MRI in 3D at $3 \mathrm{~T}$. Invest Radiol. 2012;47:252-258.

13. Ingrisch $\mathrm{M}$, Sourbron S. Tracer-kinetic modeling of dynamic contrast-enhanced MRI and CT: a primer. J Pharmacokinet Pharmacodyn. 2013;40:281-300.

14. Cohen J. Statistical Power Analysis for the Behavioral Sciences. 2nd ed. Hillsdale, NJ: Lawrence Erlbaum Associates Inc; 1988.

15. Song T, Laine AF, Chen Q, et al. Optimal k-space sampling for dynamic contrastenhanced MRI with an application to MR renography. Magn Reson Med. 2009; $61: 1242-1248$

16. Sourbron S, Biffar A, Ingrisch M, et al. PMI: platform for research in medical imaging. Magn Reson Mater Phy. 2009;22(suppl 1):539.

17. Sourbron S, Ingrisch M, Siefert A, et al. Quantification of cerebral blood flow, cerebral blood volume, and blood-brain-barrier leakage with DCE-MRI. Magn Reson Med. 2009;62:205-217.

18. R Core Team. R: A Language and Environment for Statistical Computing. Vienna, Austria: R Foundation for Statistical Computing; 2014. Available at: http://www. R-project.org. Accessed March 17, 2015.

19. Zeileis A, Hothorn T. Diagnostic checking in regression relationships. $R$ News. 2002;2(3):7-10.

20. Bates D, Mächler M, Bolker B, et al. Fitting linear mixed-effects models using lme4. J Stat Softw. 2015;67. Available at: http://www.jstatsoft.org/v67/101/. Accessed January 19, 2016.

21. Button KS, Ioannidis JP, Mokrysz C, et al. Power failure: why small sample size undermines the reliability of neuroscience. Nat Rev Neurosci. 2013;14: 365-376.

22. Larsson HB, Rosenbaum S, Fritz-Hansen T. Quantification of the effect of water exchange in dynamic contrast MRI perfusion measurements in the brain and heart. Magn Reson Med. 2001;46:272-281.

23. Paudyal R, Poptani H, Cai K, et al. Impact of transvascular and cellular-interstitial water exchange on dynamic contrast-enhanced magnetic resonance imaging estimates of blood to tissue transfer constant and blood plasma volume. J Magn Reson Imaging. 2013;37:435-444.
24. Buckley DL, Kershaw LE, Stanisz GJ. Cellular-interstitial water exchange and its effect on the determination of contrast agent concentration in vivo: dynamic contrast-enhanced MRI of human internal obturator muscle. Magn Reson Med. 2008;60:1011-1019.

25. Bains LJ, McGrath DM, Naish JH, et al. Tracer kinetic analysis of dynamic contrast-enhanced MRI and CT bladder cancer data: a preliminary comparison to assess the magnitude of water exchange effects. Magn Reson Med. 2010;64: 595-603.

26. Knutsson L, Ståhlberg F, Wirestam R. Absolute quantification of perfusion using dynamic susceptibility contrast MRI: pitfalls and possibilities. MAGMA. 2010;23:1-21.

27. Quattrocchi CC, Mallio CA, Errante Y, et al. Gadodiamide and dentate nucleus T hyperintensity in patients with meningioma evaluated by multiple follow-up contrast-enhanced magnetic resonance examinations with no systemic interval therapy. Invest Radiol. 2015;50:470-472.

28. Errante Y, Cirimele V, Mallio CA, et al. Progressive increase of T1 signal intensity of the dentate nucleus on unenhanced magnetic resonance images is associated with cumulative doses of intravenously administered gadodiamide in patients with normal renal function, suggesting dechelation. Invest Radiol. 2014;49:685-690.

29. McDonald RJ, McDonald JS, Kallmes DF, et al. Intracranial gadolinium deposition after contrast-enhanced MR imaging. Radiology. 2015;275:772-782.

30. Alsop DC, Detre JA, Golay X, et al. Recommended implementation of arterial spin-labeled perfusion MRI for clinical applications: a consensus of the ISMRM perfusion study group and the European consortium for ASL in dementia. Magn Reson Med. 2015;73:102-116.

31. Sourbron SP, Buckley DL. Tracer kinetic modelling in MRI: estimating perfusion and capillary permeability. Phys Med Biol. 2012;57:R1-R33.

32. Sourbron SP, Buckley DL. Classic models for dynamic contrast-enhanced MRI NMR Biomed. 2013;26:1004-1027.

33. Cramer SP, Simonsen H, Frederiksen JL, et al. Abnormal blood-brain barrier permeability in normal appearing white matter in multiple sclerosis investigated by MRI. NeuroImage Clin. 2014;4:182-189.

34. Cramer SP, Larsson HB. Accurate determination of blood-brain barrier permeability using dynamic contrast-enhanced T1-weighted MRI: a simulation and in vivo study on healthy subjects and multiple sclerosis patients. J Cereb Blood Flow Metab. 2014;34:1655-1665.

35. Heye AK, Thrippleton MJ, Armitage PA, et al. Tracer kinetic modelling for DCE-MRI quantification of subtle blood-brain barrier permeability. Neuroimage. 2016;125:446-455. 